\title{
Antimicrobial Release From Prefabricated Spacers Is Variable and the Dose Is Low
}

\author{
Oren Goltzer MD, Alex McLaren MD, \\ Derek Overstreet PhD, Christopher Galli BS, \\ Ryan McLemore PhD
}

Published online: 4 February 2015

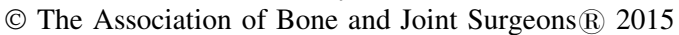

\begin{abstract}
Background High-dose antimicrobial-loaded bone cement (ALBC) is recommended to treat orthopaedic infections. Elution characteristics from prefabricated ALBC spacers and how they compare with hand-mixed ALBC are not well described. Questions/purposes (1) How does antimicrobial release from prefabricated spacers compare with release from hand-mixed ALBC over time? (2) Is antimicrobial release uniform across the surface of prefabricated ALBC spacers? (3) Do variations exist between different prefabricated spacer components? (4) Do textured surfaces release more antimicrobial than smooth surfaces?

Methods Six prefabricated ALBC spacer components, two hip and four knee, and three hand-mixed ALBC
\end{abstract}

In-kind donation by Exactech (Gainesville, FL, USA) was provided to the Orthopaedic Surgery Residency.

All ICMJE Conflict of Interest Forms for authors and Clinical Orthopaedics and Related Research ${ }^{\mathbb{B}}$ editors and board members are on file with the publication and can be viewed on request.

Clinical Orthopaedics and Related Research ${ }^{\mathbb{R}}$ neither advocates nor endorses the use of any treatment, drug, or device. Readers are encouraged to always seek additional information, including FDAapproval status, of any drug or device prior to clinical use.

O. Goltzer, A. McLaren ( $₫)$, R. McLemore

Banner Good Samaritan Medical Center, Banner

Orthopaedic Residency, 1320 North 10th Street,

Suite A, Phoenix, AZ 85006, USA

e-mail: orthopaedic.residency@bannerhealth.com

D. Overstreet

Sonoran Biosciences, 3931 West Butler Street,

Chandler, AZ 85226, USA

C. Galli

School of Biological \& Health Systems Engineering, Arizona

State University, P.O. Box 879709, Tempe, AZ 85287, USA formulations were studied in this comparative laboratory study. Gentamicin was eluted from 41 discrete sites over the surfaces of six spacer components and compared with elution from 15 ALBC specimens, five from each of three hand-mixed formulations. Elution was compared between spacer sites, components, and surface texture. Statistical analysis was performed by analysis of variance and $\mathrm{Tu}-$ key's multiple-comparison test or t-test.

Results Gentamicin release was highest in the first 24 hours for both prefabricated ALBC spacers and hand-mixed ALBC. Elution decreased over 7 days similarly for both. At Day 7 , prefabricated ALBC spacers eluted more than hand-mixed $1 \mathrm{~g}$ ALBC $(1 \mathrm{~g}$ ALBC: $1.49 \pm 0.34$, prefabricated: $3.59 \pm 1.48$, mean difference $=2.1[0.2-4.0], \mathrm{p}=0.04)$ but eluted less than $5 \mathrm{~g} \mathrm{ALBC}(9.21 \pm 1.31$, mean difference $=-5.6[-3.5$ to -7.7$], \mathrm{p}<0.001)$ and less than $10 \mathrm{~g} \mathrm{ALBC}(35.8 \pm 1.69$, mean difference $=-32.2[-29.8$ to -34.6$], \mathrm{p}<0.001$ ). Release varied from 2.7 to $9.9 \mu \mathrm{g} / \mathrm{mm}^{2}$ over the surface of the spacers and from 3.5 to $5.5 \mu \mathrm{g} / \mathrm{mm}^{2}$ between components with no component different than the others (Tukey). Release from textured surfaces was inconsistent.

Conclusions Antimicrobial release from prefabricated ALBC spacers is consistent with low-dose ALBC. Variation across the surface and between components is small compared with changes in antimicrobial load.

Clinical Relevance Antimicrobial release from prefabricated ALBC spacers is consistent with local antimicrobial delivered from other low-dose ALBC formulations.

\section{Introduction}

Orthopaedic implant infections are severe complications that result in considerable patient morbidity and cost [8]. Two-stage treatment, which is common in the United 
States $[9,22]$, typically involves removal of the implant, débridement of all infected tissues, administration of local and systemic antimicrobials, and delayed reconstruction. Local antimicrobial delivery after the débridement stage is usually from high-dose antimicrobial-loaded bone cement (ALBC) with the goal of achieving at least the minimum biofilm eradication concentration (MBEC) for the infecting microorganisms. MBEC can be hundreds of times higher than the minimum inhibitory concentration for the same organisms when they are not in biofilm, generally above the levels that would cause toxic effects systemically. When ALBC is used for local delivery in a postdébridement surgical site, it can secondarily be shaped to form a spacer that can fill dead space, and/or maintain soft tissue balance, and/or provide functional load bearing while awaiting the second-stage reconstruction [4]. Uniform release over the surface of the spacer is desirable to ensure antimicrobial delivery to all areas of the postdébridement surgical site. Some portions of the spacer may be exposed to wound/joint fluid favoring mixing and transport after release. Other areas may be closely apposed to bone with low fluid volume and low flow not favoring postrelease distribution or entrapped by bone cement used for fixation [16].

To achieve MBEC, it is generally thought that high-dose ALBC formulations are needed, leading to surgeon-directed, hand-mixed, high-dose ALBC spacers in many cases. Prefabricated ALBC spacers are available commercially with the proposed advantages of not requiring intraoperative time for fabrication of the spacer and more consistent release properties [2]. The antimicrobial powder is industrially mixed with the polymethylmethacrylate (PMMA) powder for homogeneity before polymerization. Although prefabricated spacers are made with a low-dose formulation of antimicrobial powder (1.4 wt\% gentamicin), antimicrobial release from these spacers is reported to be increased by as much as four- to fivefold, attributed to increased surface area from surface texturing on the hip femoral stems and increased permeability from new polymer technology [21]. Reported clinical outcomes using prefabricated spacers are good [18]. Antimicrobial release is expected to be uniform across the entire surface of each spacer and from spacer to spacer [19]. We therefore asked the following questions: (1) How do antimicrobial release characteristics from prefabricated spacers compare with those of hand-mixed cement over time? (2) Is antimicrobial release uniform across the surface of prefabricated ALBC spacers? (3) Do variations in release exist between different prefabricated spacer components? (4) Do the textured surfaces release more antimicrobial than the smooth surfaces?

\section{Materials and Methods}

\section{Spacer Preparation}

We used six prefabricated ALBC spacer components in all, including four knee components, one small tibia and one small femur (Interspace High Release Knee ${ }^{\circledR}$ SPK0021 with $0.9 \mathrm{~g}$ gentamicin base load), one large tibia and one large femur (Interspace High Release Knee ${ }^{\mathbb{R}}$ SPK0221 with $1.8 \mathrm{~g}$ gentamicin base load), and two hip components, one 54-mm head, short-stem (Interspace High Release Hip ${ }^{\circledR}$ SPC0122 with $1.9 \mathrm{~g}$ gentamicin base load), and one $60-\mathrm{mm}$ head, long-stem (Interspace High Release Hip ${ }^{\circledR}$ SPC0522 with 3.2 g gentamicin base load) (Tecres, Verona Italy/Exactech, Gainesville, FL, USA) (Fig. 1).

Interspace High Release implants have FDA 510K approval (K062273-2008, K101356-2011) [5, 6] based on antimicrobial release substantially equivalent to the release from spacers made with the Stage One ${ }^{\circledR}$ disposable ALBC spacer mold (Biomet, Warsaw, IN, USA) using low-dose ALBC (Cobalt G HV Bone Cement, $0.5 \mathrm{~g}$ gentamicin per 40-g batch) (Biomet) $[2,3,20]$.

To determine the amount and uniformity of antimicrobial release from surfaces of these prefabricated ALBC spacers, we formed elution wells by attaching glass tubes, 14-mm inside diameter, to the undisturbed surface of the spacer components using cyanoacrylate glue (Loctite Henkel Corp, Westlake, OH, USA) and added a waterproof barrier with epoxy (Loctite Henkel Corp, Rocky Hill, CT, USA). The ALBC surface area in each well was $154 \mathrm{~mm}^{2}$ (Fig. 2). We created 41 isolated sites distributed across the surfaces of the six spacer components. Six of these sites were on the textured surfaces of the hip component stems, three on the small-head short-stem component, and three on the large-head long-stem component (Fig. 2). A total of six spacer components were used in this study.

\section{Gentamicin Release}

Three milliliters of deionized water were placed in each elution well and kept at $37^{\circ} \mathrm{C}$. To ensure infinite sink conditions, total eluant exchange was performed on Days 1, 2, 7, and 14 [14]. Eluate was stored at $-80{ }^{\circ} \mathrm{C}$ until testing.

\section{Gentamicin Quantification}

We used a ninhydrin assay (Alfa Aesar, Heysham, Lancashire, UK) to determine the gentamicin concentration in the eluate [7]. Gentamicin concentration from each site at 

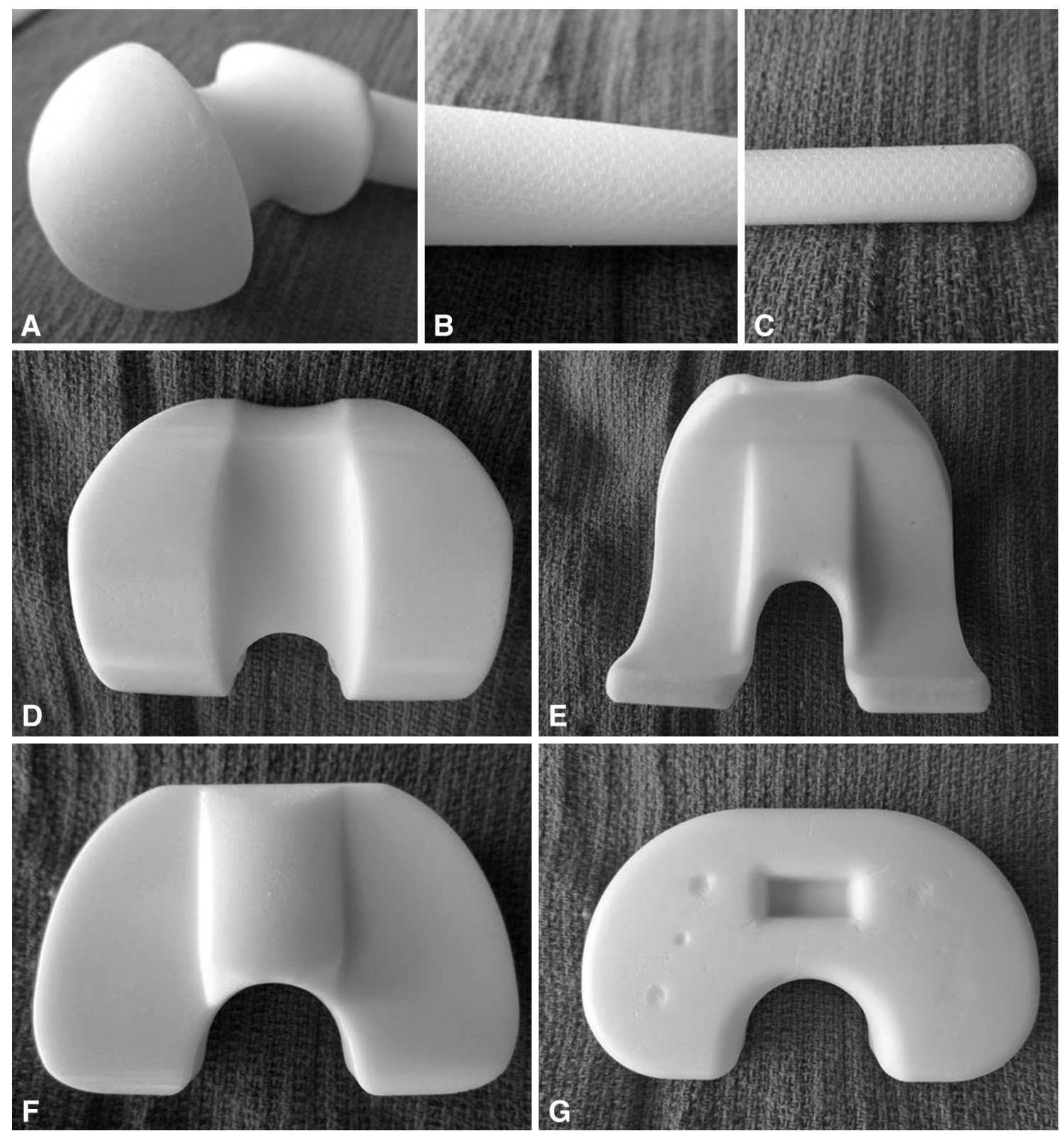

Fig. 1A-G Images of prefabricated ALBC spacers illustrate various surfaces of the spacer components that were studied. A large-head long-stem hip spacer is shown in three sections: (A) head and neck, (B) intertrochanteric region with textured surface, and (C) intramedullary stem with textured surface. The small-head, short-stem femoral spacer is similar in geometry and surface texturing but has a

each time point was determined by comparing the sample color change with a standard curve. $\mathrm{M}_{\mathrm{t}}$ (cumulative mass of recovered gentamicin at time "t") was calculated.

\section{Hand-mixed ALBC Preparation}

To determine how the antimicrobial release from the prefabricated spacer surfaces compared with release from hand- proportionately shorter intramedullary stem. A large knee spacer is shown in four images: (D) the articular surface of the femoral component, $(\mathbf{E})$ the undersurface of the femoral component, $(\mathbf{F})$ the articular surface of the tibial component, and $(\mathbf{G})$ the undersurface of the tibial component, all with smooth surfaces. The small knee spacer is similar in geometry to the small knee spacer.

mixed ALBC, three formulations of ALBC were prepared with antimicrobial content equivalent to $1 \mathrm{~g}$ (low-dose ALBC), $5 \mathrm{~g}$, or $10 \mathrm{~g}$ (high-dose ALBC) of gentamicin per $40 \mathrm{~g}$ batch of bone cement [14]. Each ALBC formulation was prepared in quarter batches: $0.25,1.25$, or $2.5 \mathrm{~g}$ gentamicin base as gentamicin sulfate (New Chemic Group, Montvale, NJ, USA) was mixed with $10 \mathrm{~g}$ of PMMA powder and polymerized with $5 \mathrm{~mL}$ of methylmethacrylate monomer (PALACOS ${ }^{\circledR}$; Zimmer, Inc, Warsaw, IN, USA). ALBC was stirred by hand using 


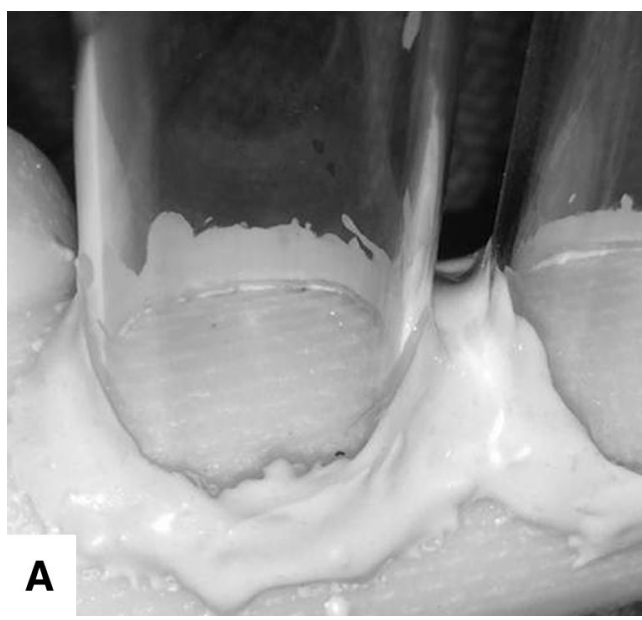

Fig. 2A-B Elution wells were formed by bonding glass tubes, $14 \mathrm{~mm}$ inside diameter, to the undisturbed surface of the prefabricated spacer components, isolating sites that were $154 \mathrm{~mm}^{2}$ in area.

a mixing bowl and spatula by an orthopaedic surgeon (AM), then while in the dough phase, it was formed into sheets approximately $2 \mathrm{~mm}$ thick by hand on a nonstick surface. After curing, hand-mixed ALBC test specimens that measured approximately $2 \mathrm{~cm} \times 2.5$ to $4.25 \mathrm{~cm} \times 2$ to $2.5 \mathrm{~mm}$ were created with a fine-toothed saw ( 32 teeth per inch) using a low cutting speed to prevent surface smearing. Five samples from each hand-mixed ALBC formulation were obtained, ranging in mass from 1.71 to $3.07 \mathrm{~g}$ and having surface areas of 10.6 to $19.8 \mathrm{~cm}^{2}$. A total of 15 hand-mixed specimens, five of each of three doses, were used in this study.

\section{Gentamicin Elution From Hand-mixed ALBC}

Hand-mixed ALBC specimens were placed in separate glass beakers and covered tightly to prevent evaporation. Twenty milliliters of phosphate-buffered saline $(150 \mathrm{mM}, \mathrm{pH} 7.4)$ were added to each beaker at $37^{\circ} \mathrm{C}$, submerging each sample completely. Total fluid exchange was performed on Days $1,3,5$, and 7 to maintain infinite sink conditions.

\section{Gentamicin Quantification for Hand-mixed ALBC}

The ninhydrin assay was used to determine the gentamicin concentration in the eluate similar to that previously described and $\mathrm{M}_{\mathrm{t}}$ was calculated.

\section{Calculation of per Surface Area Release}

$M_{t}$ is dependent on surface area. The study design required that release from undisturbed spacer surfaces was not from

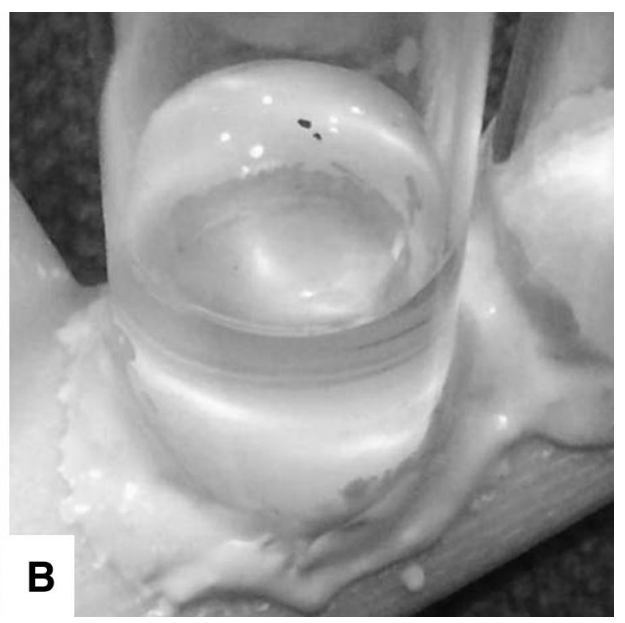

(A) The textured surface is seen a dry well on the large-head longstem hip spacer stem. (B) Eluant is seen in the well shown in A.

standardized test specimens; therefore, to compare release from one surface to another, $\mathrm{M}_{\mathrm{t}}$ for each time point and surface site was expressed per surface area $\left(\mathrm{mm}^{2}\right)$. $\mathrm{M}_{\mathrm{t}}$ was divided by the exposed surface area for either the spacer surface site or the hand-mixed ALBC specimen.

\section{Power Analysis}

We used an a priori power calculation, which assumed that sites with different release would have normally distributed variation [17]. We calculated the sample size of three to be sufficient to detect a mean difference of $80 \mu \mathrm{g}$ of drug release based on a SD within sites as large as $100 \mu \mathrm{g}$ ( $\beta=20 \%$ and $\alpha=0.05$ ).

\section{Statistical Analysis}

Comparison of the average release per surface area from all prefabricated spacer sites with the average release per surface area from all five specimens for each of the three of hand-mixed ALBC formulations was performed using t-tests. We used repeated-measures analysis of variance (ANOVA) with time, surface site, and spacer component as factors to determine differences in release per surface area from different surface sites on different preformed spacer components. Site was analyzed as an independent factor rather than a nested factor. Standard normal plots of residuals were used to show the ANOVA was well behaved [1]. We then compared between prefabricated spacer components using a Tukey's multiple comparison test. We used $\alpha=0.05$ as the cutoff for statistical significance. 


\section{Results}

Gentamicin release was highest in the first 24 hours for both prefabricated ALBC spacers and hand-mixed ALBC. Elution decreased over 7 days similarly for both. At Day 7, prefabricated ALBC spacers eluted more than hand-mixed $1 \mathrm{~g}$ ALBC ( 1 g ALBC: $1.49 \pm 0.34$, prefabricated: $3.59 \pm 1.48$, mean difference $=2.1[0.2-4.0], \mathrm{p}=0.04)$, but eluted less than $5 \mathrm{~g}$ hand-mixed ALBC $(9.21 \pm 1.31$, mean difference $=-5.6[-3.5$ to -7.7$], \mathrm{p}<0.001)$ and less than $10 \mathrm{~g}$ hand-mixed ALBC $(35.8 \pm 1.69$, mean difference $=-32.2$ [ -29.8 to -34.6$], \mathrm{p}<0.001$ ) (Table 1; Fig. 3).

For the prefabricated ALBC spacers, the amount of gentamicin released per surface area varied from site to site on Day 14 by 3.7-fold over a range from $2.7 \mu \mathrm{g} / \mathrm{mm}^{2}$ to $9.9 \mu \mathrm{g} / \mathrm{mm}^{2}$ (Fig. 4; Table 2). When analyzed across sites on Day 14, site was a determinant for release $(p=0.001)$. Four sites on the large-head long-stem hip component had higher release than the rest $(\mathrm{p}<0.001)$. The average release on Day 14 for individual prefabricated ALBC spacer components varied by $57 \%$, from 3.5 to $5.5 \mu \mathrm{g} / \mathrm{mm}^{2}$ (Fig. 4).

When analyzed across spacer component, spacer component was a determinant for release on Day $1(\mathrm{p}=0.030)$ (Fig. 5; Table 3). By Day 14, none of the implants were individually different by Tukey's test (Table 3 ).

Surface texture on the stems of the prefabricated ALBC hip spacers was not a significant determinant for release. Of the six sites on the textured surface, release from the three sites on the small-head short-stem hip component was $4.63 \mu \mathrm{g} / \mathrm{mm}^{2}$, similar to all the other sites on the other prefabricated ALBC spacer components $(\mathrm{p}=0.94)$ but lower than the release from the three sites on the largehead long-stem component, $8.99 \mu \mathrm{g} / \mathrm{mm}^{2}$ ( $\mathrm{p}=0.0011$ ). Three of the four sites with higher release were on the textured surface of the large-head long-stem hip component; one was on the nontextured surface.

\section{Discussion}

The prevailing opinion in management of orthopaedic infections is that low-dose formulations of ALBC, with antimicrobial load less than $4.5 \mathrm{~g}$ of antimicrobial powder per batch [9-11, 14], do not deliver enough antimicrobial to treat established implant and bone infection. Fragments of biofilm remain in the surgical site postdébridement necessitating MBEC throughout the entire surgical site, including the intramedullary canals. Prefabricated ALBC spacers are fabricated with a low-dose antimicrobial formulation; however, the polymer technology and surface texturing used in their fabrication are reported to increase antimicrobial release [21]. Industrial mixing is intended to

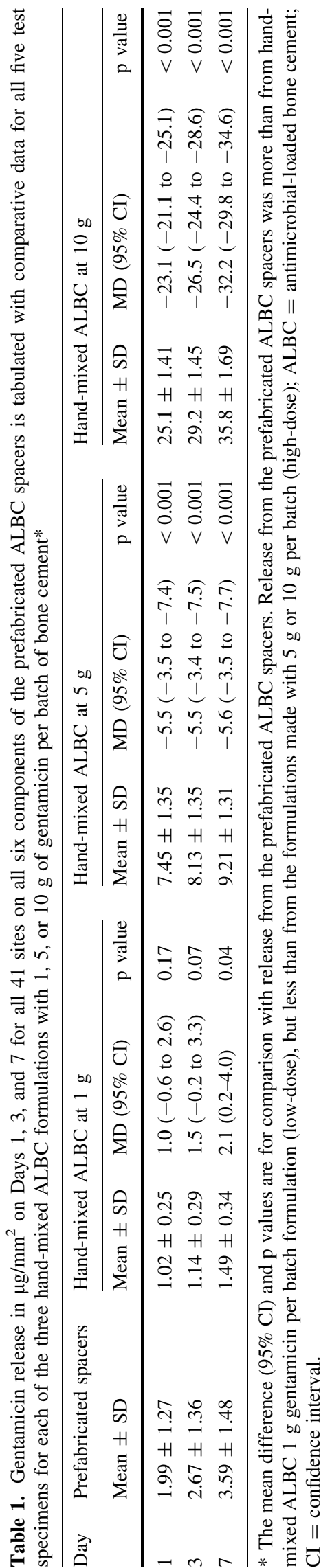




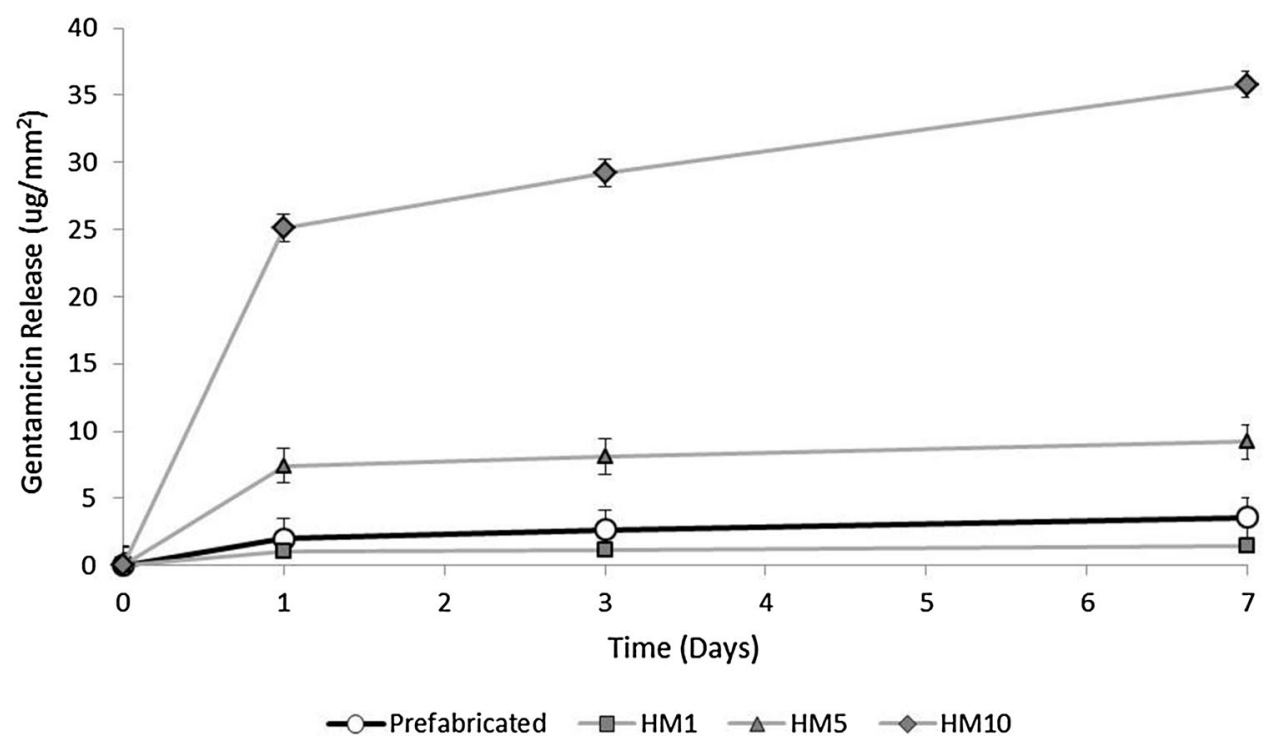

Fig. 3 Gentamicin release per $\mathrm{mm}^{2}$ on Days 1, 3 and 7, averaged over all sites measured on the prefabricated ALBC spacers is plotted with comparative data for release from three hand-mixed formulations of ALBC averaged over all five specimens for each formulation.
Release from the prefabricated ALBC spacers was more than from hand-mixed ALBC $1 \mathrm{~g}$ gentamicin per batch formulation (low-dose) but less than from the formulations made with $5 \mathrm{~g}$ or $10 \mathrm{~g}$ per batch (high-dose).

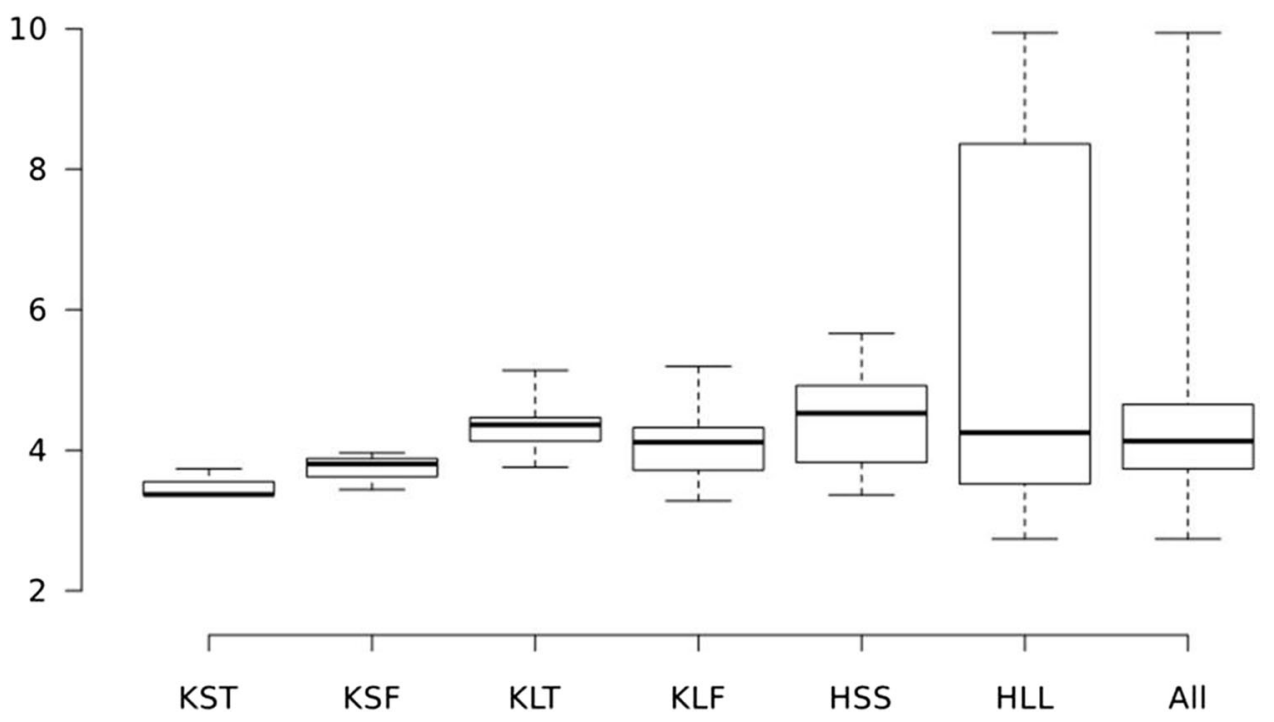

Fig. 4 The range of gentamicin release in $\mu \mathrm{g} / \mathrm{mm}^{2}$ from isolated sites on spacer surfaces by individual prefabricated ALBC spacer component, on Day 14, is represented by quartiles on the whisker-box plot. The heavy line is the median; the box represents release for $25 \%$ of the values above and below the median, and the whiskers represent

provide a high degree of homogeneity and clinical outcomes after the use of prefabricated spacers are reported to be good [19]. It is unknown how the antimicrobial release from prefabricated spacers compares with that achieved from the high-dose ALBC with $10 \mathrm{~g}$ antimicrobial powder or more per batch of cement [14]. Thus, we asked: (1) How do antimicrobial release characteristics the highest and lowest release. The categories are KST $=$ small knee tibial component; KSF $=$ small knee femoral component; KLT $=$ large knee tibial component; KLF = large knee femoral component; HSS = small-head short-stem hip spacer; HLL = large-head longstem hip spacer; ALL $=$ all spacer sites.

from prefabricated spacers compare with those of handmixed cement over time? (2) Is antimicrobial release uniform across the surface of prefabricated ALBC spacers? (3) Do variations exist in antimicrobial release between different prefabricated spacer components? (4) Do the textured surfaces release more antimicrobial than the smooth surfaces? 
Table 2. The range of gentamicin release in $\mu \mathrm{g} / \mathrm{mm}^{2}$ from isolated sites on spacer surfaces, by individual prefabricated ALBC spacer component, on Day 14, is represented in quartiles tabulated in the statistics table for the whisker-box plot (Fig. 4)*

\begin{tabular}{llllllrr}
\hline Quartile & KST & KSF & KLT & KLF & HSS & HLL \\
\hline Upper whisker & 3.73 & 3.96 & 5.14 & 5.19 & 5.66 & 9.94 & ALL \\
Third quartile & 3.55 & 3.88 & 4.46 & 4.32 & 4.92 & 8.94 \\
Median & 3.37 & 3.80 & 4.36 & 4.12 & 4.53 & 4.25 \\
First quartile & 3.36 & 3.62 & 4.13 & 3.72 & 3.83 & 3.52 & 4.13 \\
Lower whisker & 3.36 & 3.44 & 3.76 & 3.28 & 3.36 & 3.73 \\
\hline
\end{tabular}

* The categories are KST $=$ small knee tibial component; KSF = small knee femoral component; KLT = large knee tibial component; KLF = large knee femoral component; HSS = small-head short-stem hip spacer; HLL = large-head long-stem hip spacer; ALL = all spacer sites; ALBC $=$ antibiotic-loaded bone cement.

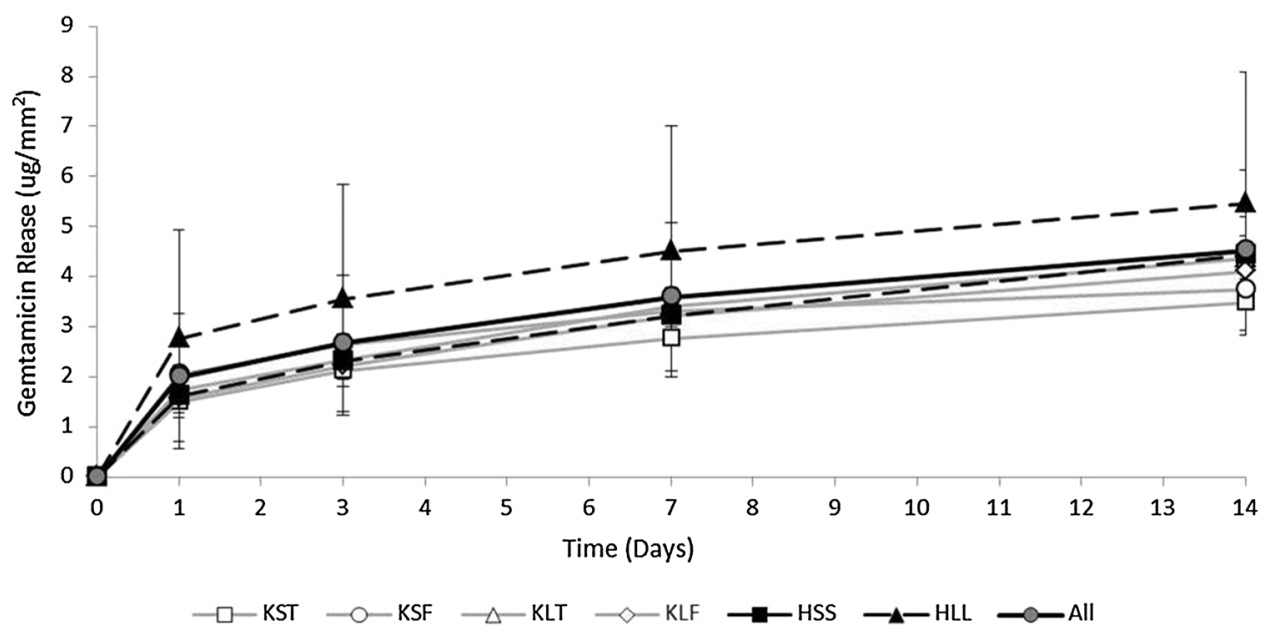

Fig. 5 Gentamicin release in $\mu \mathrm{g} / \mathrm{mm}^{2}$ on Days $1,3,7$, and 14 , by individual spacer component and combined, was highest during the first 24 hours $(\mathrm{p}=0.001)$ and accumulated at a progressively slower rate over time. Values are the mean $\pm \mathrm{SD}$ for all sites on each component or all sites on all components combined. Component was a determining factor for release by ANOVA on Day $1(\mathrm{p}=0.030)$,

There are several weaknesses of our study. First, our elution design is not standardized; however, we did maintain infinite sink conditions and data reported from other elution studies that use standardized test specimens can be expressed in release $/ \mathrm{mm}^{2}$ based on the known surface area of the standardized test specimens. Second, our methods for release from the surface of the spacer components were not identical to our methods for release from the surface of the hand-mixed ALBC. One used deionized water and the other phosphate-buffered saline; one was an isolated area on the undisturbed surface of prefabricated spacers and the other was flat, hand-shaped surfaces with narrow edges, less than $8 \%$ of the total area, that were cut with a finetooth saw using a slow nonsmearing cutting speed. The effect of deionized water versus phosphate-buffered saline on elution of water-soluble antibacterials such as gentamicin is negligible and the but by Day 14 the component was no longer a determining factor for release. The categories are $\mathrm{KST}=$ small knee tibial component; $\mathrm{KSF}=$ small knee femoral component; $\mathrm{KLT}=$ large knee tibial component; KLF = large knee femoral component; HSS = smallhead short-stem hip spacer; HLL = large-head long-stem hip spacer; $\mathrm{ALL}=$ all spacer sites .

effect that the variations in geometry have on release are also very small. Studies that use ASTM 451-08 standardized specimens generally have areas that are machined during fabrication to correct mold variations, often more than $20 \%$ of their surface. Machining causes higher burst than nonmachined surfaces, but the increased release is far less in magnitude than the effect related to changes in antimicrobial load and the effect from the machined surfaces on release is consistent throughout each study. Third, this is an in vitro study. As such it determines the antimicrobial release capability from the surfaces studied, not antimicrobial levels that will occur clinically. It is reasonable to expect that the amount of gentamicin delivered in vivo from different delivery vehicles would be proportional to the in vitro release data [13], but the actual levels that are achieved cannot be inferred from elution studies. Fourth, as an in vitro study our findings cannot be used to 
Table 3. Gentamicin release in $\mu \mathrm{g} / \mathrm{mm}^{2}$ on Day 14, by individual spacer component, is tabulated with comparison group from Tukey's multiplecomparison test*

\begin{tabular}{lllllll}
\hline Units & KST & KSF & KLT & KLF & HSS & HLL \\
\hline$\mu \mathrm{g} / \mathrm{mm}^{2}$ & $3.5 \pm 0.2$ & $3.7 \pm 0.3$ & $4.4 \pm 0.5$ & $4.1 \pm 0.6$ & $4.4 \pm 0.8$ & $5.5 \pm 2.6$ \\
Tukey & (A) & (A) & (A) & (A) & (A) & (A) \\
\hline
\end{tabular}

* Values are the mean \pm SD for all sites on each component. All spacer components are in the same Tukey comparison group indicating that they are not different. The categories are KST $=$ small knee tibial component; KSF = small knee femoral component; KLT $=$ large knee tibial component; KLF = large knee femoral component; HSS = small-head short-stem hip spacer; HLL = large-head long-stem hip spacer.

infer clinical efficacy or indications for local delivery. Our findings pertain specifically to the antimicrobial release capabilities of the specific prefabricated ALBC spacers and hand-mixed ALBC formulations that we studied. How those capabilities are integrated into a treatment plan is a clinical decision specific to each patient.

We found that gentamicin release from both the prefabricated ALBC spacers and the hand-mixed ALBC was highest initially but then slowed after the first day. Our findings are consistent with previously reported release data from ALBC $[12,23]$. The amount of gentamicin that is released from the prefabricated ALBC spacer surfaces is more than we measured from $1 \mathrm{~g} / \mathrm{batch}$ ALBC, but less than we measured from higher antimicrobial loads of $5 \mathrm{~g}$ or $10 \mathrm{~g}$ per batch of hand-mixed ALBC. The higher release compared with the hand-mixed $1 \mathrm{~g}$ ALBC formulation may be explained by the polymer technology used by the manufacturer. We would also expect the release from prefabricated ALBC spacers to be more than the release from the surfaces of other commercially premixed ALBC formulations with 0.5 to $1 \mathrm{~g}$ of antimicrobial per batch [15]; however, neither the prefabricated ALBC spacers nor hand-mixed ALBC with $1 \mathrm{~g}$ antimicrobial per batch are capable of releasing amounts of antimicrobial equivalent to the amounts released from higher dose formulations at either $5 \mathrm{~g}$ or the $10 \mathrm{~g}$ loads per batch.

Gentamicin release from the surface of the prefabricated ALBC spacers varied across the surfaces, site to site, by up to $263 \%$ and from spacer to spacer by up to $57 \%$. Prefabricated ALBC spacers are often placed in sites with large variations in the gap between bone and the spacer with the potential for wide variations in fluid volume and flow, which is important for antimicrobial transport to the rest of the surgical site. Also, prefabricated ALBC spacers are often fixed with higher dose ALBC, which adds antimicrobial to that released from the prefabricated spacer surfaces [24] but isolates some regions of the spacer surface from others. The net effect on antimicrobial levels in various regions of the postdébridement surgical site has yet to be measured. Although the variation in gentamicin release from the surfaces of prefabricated ALBC spacers is measurable, it is not as important as the 10-fold difference between low-dose and high-dose ALBC formulations. The methods we used to study antimicrobial release from the surface of prefabricated spacers are applicable to any other surface that provides antimicrobial release and may be useful for future comparative studies.

For the textured surface, there was a twofold difference in gentamicin released from the textured surfaces between the two components we studied. There was no difference in release between the nontextured surfaces on all the knee components and the textured surface on the small-head short-stem hip component, and one of the nontextured surface sites on the large-head long-stem hip components had similar release to the textured surface on that component. We expected to find an increase in release from textured surfaces as a result of the increased surface area caused by the texturing. However, our data are not consistent with any pattern of effect that may be the result of the texturing.

Gentamicin release from prefabricated ALBC spacers is consistent with low-dose hand-mixed ALBC formulations with less than $4.5 \mathrm{~g}$ of antimicrobial powder per batch. Although variations in release across their surfaces can be measured, these variations are small compared with the difference between low-dose and high-dose ALBC and are not specific to surface texturing. Clinical outcomes for treatment of implant and bone infection are dependent on more factors than local antimicrobial delivery. Other factors, including surgical débridement and host immune status, play important roles. When local delivery is used, the release capability of the local depot is important information. Local antimicrobial delivery from prefabricated ALBC spacers may be indicated in cases where low-dose ALBC formulations are considered appropriate.

\section{References}

1. Altman DG. Practical Statistics for Medical Research. 2nd ed. Boca Raton, FL, USA: Chapman \& Hall/CRC; 2006.

2. Bertazzoni Minelli E, Benini A, Magnan B, Bartolozzi P. Release of gentamicin and vancomycin from temporary human hip 
spacers in two-stage revision of infected arthroplasty. $J$ Antimicrob Chemother. 2004;53:329-334.

3. Bertazzoni Minelli E, Della Bora T, Benini A. Different microbial biofilm formation on polymethylmethacrylate (PMMA) bone cement loaded with gentamicin and vancomycin. Anaerobe. 2011;17:380-383.

4. Cohen JC, Hozack WJ, Cuckler JM, Booth REJ. Two-stage reimplantation of septic total knee arthroplasty. Report of three cases using an antibiotic-PMMA spacer block. J Arthroplasty. 1988;3:369-377.

5. Food and Drug Administration. 510(k) Summary K062273 SpacerG Modification. 208AD. Available at: www.accessdata.fda.gov/ cdrh_docs/pdf6/K062273.pdf. Accessed January 15, 2015.

6. Food and Drug Administration. 510(k) Summary K101356. 2011. Available at: www.accessdata.fda.gov/scripts/cdrh/cfdocs/cfPMN/ pmn.cfm?ID=K101356. Accessed January 16, 2015.

7. Frutos P, Torrado S, Perez-Lorenzo ME, Frutos G. A validated quantitative colorimetric assay for gentamicin. J Pharm Biomed Anal. 2000;21:1149-1159.

8. Gaine WJ, Ramamohan NA, Hussein NA, Hullin MG, McCreath $\mathrm{SW}$. Wound infection in hip and knee arthroplasty. J Bone Joint Surg Br. 2000;82:561-565.

9. Hanssen AD, Spangehl MJ. Practical applications of antibioticloaded bone cement for treatment of infected joint replacements. Clin Orthop Relat Res. 2004;427:79-85.

10. Jiranek W. Antibiotic-loaded cement in total hip replacement: current indications, efficacy, and complications. Orthopedics. 2005;28:s873-877.

11. Lautenschlager EP, Marshall GW, Marks KE, Schwartz J, Nelson CL. Mechanical strength of acrylic bone cements impregnated with antibiotics. J Biomed Mater Res. 1976;10:837-845.

12. Marks KE, Nelson CL, Lautenschlager EP. Antibiotic-impregnated acrylic bone cement. J Bone Joint Surg Am. 1976;58:358-364.

13. McLaren A, Giers MB, Fraser J, Hosack L, Caplan MR, McLemore R. Antimicrobial distribution from local delivery depends on dose: a pilot study with MRI. Clin Orthop Relat Res. 2014;472:3324-3329.

14. McLaren A, Gutierrez F, Martin M, McLemore R. Local antimicrobial treatment. In: Cierny G III, McLaren AC,
Wongworawat MD, eds. Orthopaedic Knowledge Update: Musculoskeletal Infection. 1st ed. Rosemont IL, USA: AAOS; 2009:95-116.

15. McLaren A, Nugent M, Economopoulos K, Kaul H, Vernon BL, McLemore R. Hand-mixed and premixed antibiotic-loaded bone cement have similar homogeneity. Clin Orthop Relat Res. 2009;467:1693-1698.

16. Moojen DJF, Hentenaar B, Charles Vogely H, Verbout AJ, Castelein RM, Dhert WJA. In vitro release of antibiotics from commercial PMMA beads and articulating hip spacers. $J$ Arthroplasty. 2008;23:1152-1156.

17. Ott RL, Longnecker MT. An Introduction to Statistical Methods and Data Analysis. 6th ed. Independence, KY, USA: Cengage Learning; 2008.

18. Pattyn C, De Geest T, Ackerman P, Audenaert E. Preformed gentamicin spacers in two-stage revision hip arthroplasty: functional results and complications. Int Orthop. 2011;35:1471-1476.

19. Regis D, Sandri A, Samaila E, Benini A, Bondi M, Magnan B. Release of gentamicin and vancomycin from preformed spacers in infected total hip arthroplasties: measurement of concentrations and inhibitory activity in patients' drainage fluids and serum. ScientificWorldJournal. 2013;2013:752184.

20. Romanò CL, Romanò D, Logoluso N, Meani E. Septic versus aseptic hip revision: how different? J Orthop Traumatol. 2010;11:167-174.

21. Soffiatti R. The preformed spacers: from the idea to the realization of an industrial device. In: Meani E, Romanò C, Crosby L, Hofmann G, eds. Infection and Local Treatment in Orthopedic Surgery. Berlin, Germany: Springer-Verlag; 2007:201-204.

22. Tetsworth K. Infection after total knee arthroplasty: evaluation and treatment. Curr Opin Orthop. 2003;14:45-51.

23. Wahlig H, Dingeldein E. Antibiotics and bone cements. Experimental and clinical long-term observations. Acta Orthop Scand. 1980;51:49-56.

24. Wan Z, Karim A, Momaya A, Incavo SJ, Mathis KB. Preformed articulating knee spacers in 2-stage total knee revision arthroplasty: minimum 2-year follow-up. J Arthroplasty. 2012; 27:1469-1473. 